\title{
Outbreak of Human Metapneumovirus in Ibaraki, Japan and Its Descriptive Epidemiology
}

\author{
Junko Kurita', Natsuki Nagasu' ${ }^{2}$, Noriko Nagata ${ }^{3}$, Hideo Okuno ${ }^{4}$, \\ Tamie Sugawara ${ }^{5}$, Yasushi Ohkusa ${ }^{5}$
}

\author{
${ }^{1}$ The Graduate School of Health Sciences, Ibaraki Prefectural University, Ibaraki, Japan \\ ${ }^{2}$ Health Services Disease Control Division, Department of Health and Social Services, Ibaraki, Japan \\ ${ }^{3}$ Public Health Research Center, Ibaraki, Japan \\ ${ }^{4}$ Medical School, Osaka University, Osaka, Japan \\ ${ }^{5}$ National Institute of Infectious Diseases, Tokyo, Japan \\ Email: tammy@nih.go.jp
}

How to cite this paper: Kurita, J., Nagasu, N., Nagata, N., Okuno, H., Sugawara, T. and Ohkusa, Y. (2018) Outbreak of Human Metapneumovirus in Ibaraki, Japan and Its Descriptive Epidemiology. Health, 10, 749-757. https://doi.org/10.4236/health.2018.106057

Received: May 11, 2018

Accepted: June 17, 2018

Published: June 20, 2018

Copyright $\odot 2018$ by authors and Scientific Research Publishing Inc. This work is licensed under the Creative Commons Attribution International License (CC BY 4.0).

http://creativecommons.org/licenses/by/4.0/

\section{c) (i) Open Access}

\begin{abstract}
Introduction: The Infection Control Law in Japan does not cover monitoring of human metapneumovirus (HMPV). Therefore, its epidemiology is not well known. However, rapid diagnostic testing for this virus has been reimbursed by public health insurance in Japan since 2014. One case of acute encephalitis caused by HMPV was reported in April 2017 in Ibaraki. All schools in Ibaraki prefecture started to participate in the (Nursery) School Absenteeism Surveillance System ((N)SASSy) in 2009. All nursery schools started to use it in 2012. Methods: We specifically examine its seasonality and incidence by age. The study period extends from October 1, 2014 through May 31, 2017. Results: In total, (N)SASSy received 187 cases during the period. Spring and early summer were apparently the high season. The highest incidence was 36 cases in April 2015. The incidence among zero and one year olds was higher in 2017 than in the same season among 2014-2016. The necessary effort to download data from (N)SASSy, show these figures, and confirm the situation and background was a few minutes. Discussion: Rapid diagnostic testing for HMPV elucidated its epidemiology for the first time. Moreover, timely information sharing through (N)SASSy led the Ibaraki prefectural office to publish a reminder in July 2017 about the HMPV outbreak situation and to encourage precautions against it by nursery schools.
\end{abstract}

\section{Keywords}

Human Metapneumovirus, Infection Control Law, (Nursery) School Absenteeism Surveillance System, Surveillance 


\section{Introduction}

An outbreak of human metapneumovirus (HMPV), a virus usually causing respiratory symptoms such as pneumonia or bronchitis among children [1] [2] [3], was reported at a facility for elderly people in Japan in 2013 [4]. Elderly people and infants are at high risk for complications from HMPV. Therefore, more attention must be devoted to treating them [5]. Although many infants are reportedly infected by this virus, similarly to respiratory syncytial virus (RS virus) infection, because national official surveillance based on the Infection Control Law does not cover monitoring of this disease, its epidemiology has remained unclear. Even outside of Japan, although some epidemiological studies [6]-[13] have been conducted, these were retrospective and serological studies based on analyses of specimens collected from influenza-like-illness (ILI) patients by RT-PCR. Those studies found that the high season of HMPV has been late winter or early spring [7] [10] [11] [12] [13]. Nevertheless, these studies were neither prospective nor comprehensive. The studies included neither sampling nor coverage of all HMPV patients in the community. More importantly, rapid diagnostic testing and information sharing systems covering the community are lacking.

Rapid diagnostic testing for HMPV has been reimbursed by public health insurance in Japan since 2014. Consequently, physicians can diagnose cases as HMPV infection. Caregivers notify the children's (nursery) schools of this diagnosis when they are absent. The term of "(nursery) school" includes both of nursery school and school.

The (Nursery) School Absenteeism Surveillance System ((N)SASSy) uses Japan's own unique (nursery) school culture. Caregivers of students who are absent from (nursery) school because of infectious diseases usually report to the (nursery) school. In the case of HMPV, if the students or children are diagnosed as having HMPV, then their caregivers are obligated to notify the (nursery) school. School nurses or other teachers provide information to (N)SASSy via the internet. Registration of diseases occurs at once for one episode. Therefore, duplication for the same students never occurs in the same school year. In another school year, registration of the same disease for the same student is possible. As of the end of 2016, the system covered approximately 37,000 schools, including 10,000 nursery schools, or about $60 \%$ of all schools and $40 \%$ of all nursery schools in Japan. On a daily basis, it monitors the health condition of about four million children younger than 18 years old. The (N)SASSy information is used by (nursery) schools themselves to recognize the situation in surrounding areas and to inform teachers, students, and caregivers, thereby promoting precautionary measures if some outbreaks are reported in the surrounding areas but not at their own (nursery) school. The (N)SASSy information is also shared among (nursery) school doctors, educational boards, local government offices for nursery schools, public health centers, and local medical associations. That shared information encourages earlier awareness of infectious diseases, which engend- 
ers earlier responses to the initial stage of outbreaks in (nursery) schools. Moreover, having such a large trove of data related to children's health facilitates large epidemiological studies. It has been developed by the research group headed by Dr. Ohkusa, one of the authors of this paper, and has been keeping its copyright. Currently, the system is operated by the Japanese Society of School Health.

Ibaraki prefecture, located near metropolitan Tokyo, has about three million total population and 0.46 million persons younger than 18 years old. All schools started to participate in (N)SASSy in 2009 and all nursery schools started to use it in 2012 [14] [15] [16] [17] [18]. In 2014, it became possible to monitor the health condition of all children from 0 to 18 years old in (nursery) schools in a timely manner. It has been used for some countermeasures against influenza, measles, and rubella by the local government and public health center in Ibaraki [19], but no reports have described studies of HMPV.

Patients of acute encephalitis/encephalopathy were monitored by national official surveillance based on the Infection Control Law in April 2017. Therefore, physicians who diagnose acute encephalitis/encephalopathy are obligated to report it to the public health center for whatever virus causes it. In April 2017, one acute encephalopathy associated with HMPV was reported in Ibaraki [20] among 26 cases reported during the year through March 2017 in Ibaraki [21] [22] [23]. This is the first case of acute encephalopathy associated with an HMPV case in Ibaraki. Nevertheless, it was a severe case of HMPV. The prefectural government office must ascertain the epidemiology of HMPV as background information. As described above, national official surveillance based on the Infection Control Law does not cover the monitoring of HMPV itself, but its epidemiological aspects have remained unclear. We cannot assess its situation without (N)SASSy, even at present. Therefore, we conducted the study to evaluate the epidemic situation of HMPV using (N)SASSy and possible public health responses. It might be the first prospective and comprehensive study for HMPV in the community.

This paper was composed to present a descriptive epidemiology of the HMPV infection, especially by month or by age in the (nursery) school children using (N)SASSy, rather than serological study. Moreover, we were motivated to demonstrate the usefulness of (N)SASSy at (nursery) schools and for public health offices and local government through this experience.

\section{Methods}

As of April 2017 in Ibaraki, 572 nursery schools and 1172 schools participate in (N)SASSy, providing data of 60,236 children in nursery schools and 309,742 students in schools. The period of the present study extended from October 1, 2014 through May 31, 2017.

Data were obtained from (N)SASSy by the Health Services Disease Control Division, Department of Health and Social Services, Ibaraki prefecture. Incidence was defined as the number of reported cases divided by the number of all 
students in the same school age at the end of the month or school year. We specifically examined seasonality and incidence by age through descriptive epidemiology using comparison with April 2017, when acute encephalitis was reported. We also examined incidence in April and May of each year to evaluate its background.

In addition, statistical tests using chi-square tests were applied if we found some difference. We adopted $5 \%$ as significance level.

\section{Ethical Considerations}

(N)SASSy includes only anonymous data that have been de-linked from individual patient information with no private information such as a name, address or phone number. Moreover, we used only the counted number of students. Therefore, ethical issues in Japan related to the epidemiological study were not applicable to this study. For that reason, no formal ethical review was required.

\section{Results}

In total, (N)SASSy received 187 cases for the period. Figure 1 depicts the number of patients with HMPV in Ibaraki by month from September 2014. Results suggest that spring and early summer constitute a high season. The highest incidence was 36 children in April 2015, followed by 27 children in April 2017, and 20 children in April 2014. In 2016, the number of patients was highest in June, not April.

Figure 2 depicts the incidence of HMPV per 1000 populations in Ibaraki by age in April and May of 2014, 2015, 2016, and 2017. Incidences among zero and one year olds were higher in 2017 than in the same season among 2014-2016. Especially, for zero-year-old children, the incidence was more than two times higher in 2017 than it had been earlier. For one-year-old children, it was higher by more than 0.3 per 1000 population than before. In April, 2015, when the greatest number of patients was identified as shown in Figure 1, the incidence of two-year-old children was higher than in other years, as shown in Figure 2, even though the highest incidence of zero or one-year-old children was in April and May, 2017. We applied statistical tests to incidence rates of zero or one-year-old children in April and May in 2017 and earlier. The respective obtained $p$-values were 0.0379 and 0.0859 . Therefore, even though the incidence rate of zero-year-old children in 2017 was significantly higher than before, the incidence rate among one-year-old children was not significantly different from 2017 and before. Moreover, statistical tests for incidence rates of two-year-old children in April and May in 2015 and other years showed 0.0451 as the $p$-value. Consequently, the incidence of two-year-old children in April and May, 2015 was not significantly higher than in other years.

From the Health Services Disease Control Division, Department of Health and Social Services, Ibaraki prefecture, data downloaded from (N)SASSy, during several minutes show these figures and confirm the situation and background. 


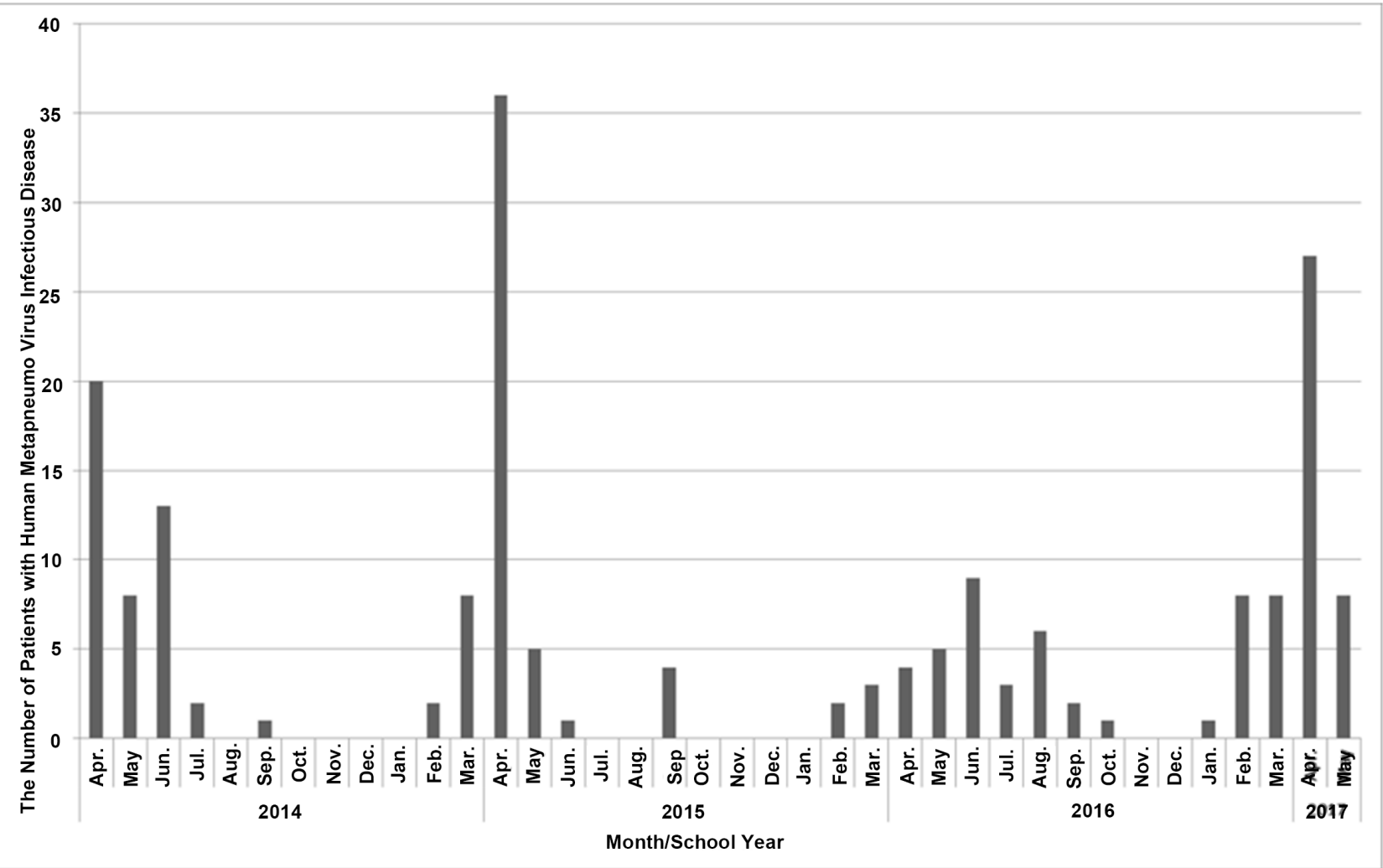

Figure 1. Number of patients with HMPV in Ibaraki by month from September 2014.

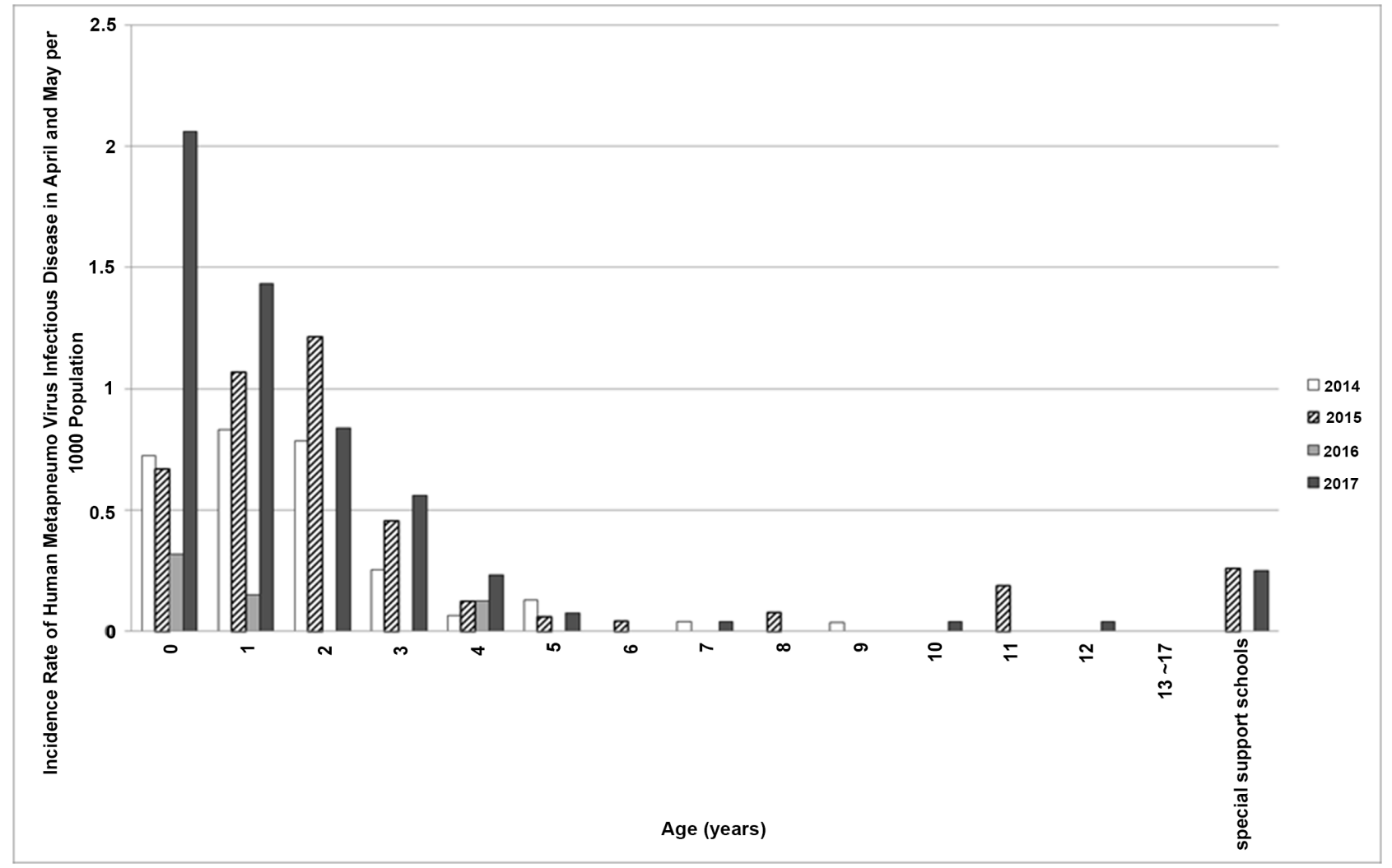

Figure 2. Incidence of HMPV per 1,000 population in Ibaraki by age in April and May of 2014, 2015, 2016, and 2017. 


\section{Discussion}

Although HMPV has sometimes been identified from samples in the outbreak of respiratory symptoms to date [6]-[13], its epidemiology has remained unclear, including information related to situations when large outbreaks did not occur. Incidence rates in April and May of 2015 and 2017 were higher, especially among zero and one year olds. Infants usually have a narrow nasal tract, choanae, and air passage. Therefore, caregivers of infants must know the background and prevent this disease.

In addition, although influenza virus, RS virus, parainfluenza virus, coronavirus, rhinovirus, and adenovirus cause respiratory symptoms in infants, many viruses could not be diagnosed at bedside, except for influenza and RS virus, until 2014. However, because rapid diagnostic testing for HMPV was approved in 2014, bedside diagnosis has become possible recently. These rapid diagnostic tests enable us to make timely and earlier responses to prevent severe outbreaks at (nursery) schools [19].

The epidemiology of HMPV has remained unclear because national official surveillance based on the Infection Control Law does not cover it. However, since 2014, caregivers report diagnosed HMPV infection to (nursery) schools when children are absent. Then (nursery) schools register this information to (N)SASSy. In such cases, the epidemiology of HMPV became apparent for the first time. Especially, the incidence was found to be higher in April and May of 2017. Therefore, we consider that a reminder to take precautions is necessary for caregivers to avoid severe and complex cases. In fact, Ibaraki prefecture office published a reminder about the HMPV outbreak situation and prevention methods for (nursery) schools in July, 2017. The (nursery) schools receiving such information were apprised of the situation in a timely manner. They detected the outbreak earlier in their (nursery) schools, explained it, and applied prevention methods more appropriately to caregivers.

Three seasons can be compared because all (nursery) schools joined (N)SASSy in 2014 in Ibaraki. Such comprehensive participation over a long period is quite useful for timely, prefecture-wide situation awareness and investigation or study. In this case, for HMPV, acute encephalopathy associated with HMPV in April 2017 strongly motivated the Health Services Disease Control Division, Department of Health and Social Services, Ibaraki prefecture to ascertain the situation and epidemiology. (N)SASSy can provide necessary information in a few minutes and can then promote timely reminders to (nursery) schools and caregivers.

To date, the high season of HMPV has been late winter or early spring [7] [10] [11] [12] [13], which were result of serological studies based on specimens collected from ILI patients by RT-PCR. However, the present study revealed that April and May are the high season, but not winter. Our scheme is much more comprehensive for school children based on rapid test results that are not limited to ILI patients. In winter, because there were numerous ILI patients, a 
larger number of specimens than in other seasons were tested, revealing a larger number of HMPV positive cases than in earlier studies. It might be misleading because of the biased sampling scheme. However, our scheme was unaffected by the outbreak of influenza. Conversely, our scheme was limited to (nursery) school children. April is the first month in the school year in Japan, even at nursery schools. Therefore, in April many newcomers of age zero or those who have never experienced group life join nursery schools, presenting many chances of contact with other children. This unique phenomenon might raise activity of HMPV in Japan. Therefore, our finding that April and May are the high season of HMPV might be a phenomenon that is unique to Japan.

An important limitation of this study was the exclusion of children who did not attend nursery schools or kindergartens: (N)SASSy did not cover those children. Those children might have lower incidence because they do not engage in communal living. Therefore, the opportunities of contact with patients might be fewer than for children in nursery schools or kindergarten. If so, our estimation for incidence shows an apparent upward bias. Moreover, (N)SASSy is based on information from caregivers to (nursery) schools. Although the diagnosis of HMPV depends on rapid diagnosis test results, caregivers might misunderstand or misreport results to (nursery) schools. The latter is an apparent limitation of (N)SASSy itself, not of this research.

Nevertheless, results of this study are expected to be valuable for initial examination of the epidemiology of HMPV in Japan. The results can promote a policy of notifying (nursery) schools and caregivers of an impending risk of infection.

However, because (N)SASSy does not cover elderly facilities, we cannot ascertain situations of HMPV or other diseases at those facilities in a few minutes and then publish a timely reminder. Covering such facilities presents a challenge for our future research.

\section{Conclusion}

We demonstrated that, using (N)SASSy, public health offices or local governments can ascertain the epidemiology of HMPV in a few minutes and can provide a timely reminder to (nursery) schools and caregivers. (N)SASSy can also detect outbreaks earlier and make timely responses, thereby improving public health even for diseases that are not monitored under the Infection Control Law such as HMPV. We would like to continue timely evaluation for infectious diseases using (N)SASSy and to promote a policy of reminding at-risk populations to take precautions.

\section{Acknowledgements}

This research is a part of outcome form Grant-in-Aid for Scientific Research (C) 15K01676 "Research for guidance and effort for introduction of (nursery) school absenteeism surveillance system" headed by Tamie Sugawara, senior scientist, national Institute of Infectious Diseases. 


\section{References}

[1] Peiris, J.S., Tang, W.H., Chan, K.H., et al. (2003) Children with Respiratory Disease Associated with Metapneumovirus in Hong Kong. Emerging Infectious Diseases, 9, 628-633. https://doi.org/10.3201/eid0906.030009

[2] van den Hoogen, B.G., de Jong, J.C., Groen, J., van den Hoogen, et al. (2001) A Newly Discovered Human Pneumovirus Isolated from Young Children with Respiratory Tract Disease. Nature Medicine, 7, 719-724. https://doi.org/10.1038/89098

[3] Wolf, D.G., Greenberg, D., Kalkstein, D., et al. (2006) Comparison of Human Metapneumovirus, Respiratory Syncytial Virus and Influenza A Virus Lower Respiratory Tract Infections in Hospitalized Young Children. The Pediatric Infectious Disease Journal, 25, 320-324. https://doi.org/10.1097/01.inf.0000207395.80657.cf

[4] Yokoi, H., Mizumura, A., et al. (2013) Outbreak of Human Metapneumovirus Infection in a Welfare Facility, April 2013-Chiba City. Infectious Agents Surveillance Report, 34, 402. (In Japanese)

[5] Boivin, G., Abed, Y., Pelletier, G., et al. (2002) Virological Features and Clinical Manifestations Associated with Human Metapneumovirus: A New Paramyxovirus Responsible for Acute Respiratory-Tract Infections in All Age Groups. The Journal of Infectious Diseases, 186, 1330-1334. https://doi.org/10.1086/344319

[6] Reiche, J., Jacobsen, S., Neubauer, K., Hafemann, S., Nitsche, A., Milde, J., Wolff, T. and Schweiger, B. (2014) Human Metapneumovirus: Insights from a Ten-Year Molecular and Epidemiological Analysis in Germany. PLoS One, 9, e88342. https://doi.org/10.1371/journal.pone.0088342

[7] Stockton, J., Stephenson, I., Fleming, D., et al. (2002) Human Metapneumovirus as a Cause of Community-Acquired Respiratory Illness. Emerging Infectious Diseases, 8, 897-901. https://doi.org/10.3201/eid0809.020084

[8] Rafiefard, F., Yun, Z. and Orvell, C. (2008) Epidemiologic Characteristics and Seasonal Distribution of Human Metapneumovirus Infections in Five Epidemic Seasons in Stockholm, Sweden, 2002-2006. Journal of Medical Virology, 80, 1631-1638. https://doi.org/10.1002/jmv.21242

[9] Gioula, G., Chatzidimitriou, D., Melidou, A., et al. (2010) Contribution of Human Metapneumovirus to Influenza-Like Infections in Northern Greece, 2005-2008. Eurosurveillance, 15, pii: 19499. https://doi.org/10.2807/ese.15.09.19499-en

[10] Williams, J.V., Harris, P.A., Tollefson, S.J., Halburnt-Rush, L.L., Pingsterhaus, J.M., Edwards, K.M., Wright, P.F. and Crowe Jr., J.E. (2004) Human Metapneumovirus and Lower Respiratory Tract Disease in Otherwise Healthy Infants and Children. The New England Journal of Medicine, 350, 443-450. https://doi.org/10.1056/NEJMoa025472

[11] Jartti, T., Lehtinen, P., Vuorinen, T., Osterback, R., van den Hoogen, B., Osterhaus, A.D. and Ruuskanen, O. (2004) Respiratory Picornaviruses and Respiratory Syncytial Virus as Causative Agents of Acute Expiratory Wheezing in Children. Emerging Infectious Diseases, 10, 1095-1101. https://doi.org/10.3201/eid1006.030629

[12] Bosis, S., Esposito, S., Niesters, H.G., Crovari, P., Osterhaus, A.D. and Principi, N. (2005) Impact of Human Metapneumovirus in Childhood: Comparison with Respiratory Syncytial Virus and Influenza Viruses. Journal of Medical Virology, 75, 101-104. https://doi.org/10.1002/jmv.20243

[13] Gerna, G., Campanini, G., Rovida, F., Sarasini, A., Lilleri, D., Paolucci, S., Marchi, A., Baldanti, F. and Revello, M.G. (2005) Changing Circulation Rate of Human Metapneumovirus Strains and Types among Hospitalized Pediatric Patients during Three Consecutive Winter-Springs. Brief Report. Archives of Virology, 150, 
2365-2375. https://doi.org/10.1007/s00705-005-0581-2

[14] Shimatani, N., Sugishita, Y., Sugawara, T., Nakamura, Y., Ohkusa, Y., Yamagishi, T., et al. (2015) Enhanced Surveillance for the Sports Festival in Tokyo 2013: Preparation for the Tokyo 2020 Olympic and Paralympic Games. Japanese Journal of Infectious Diseases, 68, 288-295. https://doi.org/10.7883/yoken.JJID.2014.233

[15] Suzue, T., Hoshikawa, Y., Nishihara, S., Fujikawa, A., Miyatake, N., Sakano, N., et al. (2012) The New School Absentees Reporting System for Pandemic Influenza A/H1N1 2009 Infection in Japan. PLoS One, 7, e30639. https://doi.org/10.1371/journal.pone.0030639

[16] Ohkusa, Y., Sugawara, T., Mitani, M., Sugiura, H. and Okabe, N. (2011) Development and Evaluation of a School Absenteeism Reporting System. Journal of School Health, 53, 312-319. (In Japanese)

[17] Sugawara, Y., Fujimoto, T., Ohkusa, Y., Sugishita, Y., Konagaya, M., Sugiura, H., Taniguchi, K. and Okabe, N. (2012) The Possibility of Outbreak Control by Real-time Surveillance with PCR Method Performed Immediately-A Case Study of Hand Foot and Mouth Disease Outbreak in a Day Care Facility for Children. The Journal of the Japanese Association for Infectious Diseases, 86, 405-410. (In Japanese)

[18] Sugawara, T. and Ohkusa, Y. (2013) Two Aspects of Infectious Disease Surveillance at School and Nursery School: Early Response Based on Early Detection and Recognition of Incidence of Infectious Diseases from Nursery School Kids to High School Students. The Journal of Child Health, 72, 610-612. (In Japanese)

[19] Watanabe, M., Kurita, J., Takagi, T., Nagata, N., Nagasu, N., Sugawara, T. and Ohkusa, Y. (2016) Early Detection and Response for Measles and Rubella Cases through the (Nursery) School Absenteeism Surveillance System in Ibaraki Prefecture. Japanese Journal of Public Health, 63, 209-214. (In Japanese)

[20] Infectious Disease Surveillance Center, National Institute of Infectious Disease, the Ministry of Health, Labour and Welfate, Infectious Disesas Weekly Rreport on the 17th and 18th Week, 2017. (In Japanese) https://www0.nih.go.jp/niid/idsc/idwr/IDWR2017/idwr2017-17-18.pdf

[21] Ibaraki Infectious Disease Surveillance Center, Infectious Disease Weekly Report Ibaraki on the 13th Week, 2016. (In Japanese)

http://www.pref.ibaraki.jp/hokenfukushi/eiken/idwr/weekly/documents/2016idwr1 3.pdf

[22] Ibaraki Infectious Disease Surveillance Center, Infectious Disease Weekly Report Ibaraki on the 52nd Week, 2016. (In Japanese)

http://www.pref.ibaraki.jp/hokenfukushi/eiken/idwr/weekly/documents/2016idwr5 2.pdf

[23] Ibaraki Infectious Disease Surveillance Center Infectious Disease Weekly Report Ibaraki on the 13th Week, 2017. (In Japanese)

http://www.pref.ibaraki.jp/hokenfukushi/eiken/idwr/weekly/documents/2017idwr1 3.pdf 